\title{
Numerical Simulation of Viscous Effects of Oil on the Capacity of Oil Booms
}

\author{
Thai Thi Kim Chi* \\ University of Transport and Communications, Urban Transport and Coastal Engineering Department, Hanoi, Vietnam
}

\begin{abstract}
The potential risk of oil spills is constantly increasing, which required special techniques, equipment and effective response plans as well. Oil booms is the most common equipment used for spilled oil clean-up. However, due to different reasons such as extreme hydrological conditions, unreasonable design or poor handling, the failure of booms can happen. The loss rate of boom is acceptable if it is small enough. In the present paper, the behavior of oil with different viscosity around the oil boom is numerically studied using ANSYS FLUENT software. The shape evolution of oil slicks is presented. The loss rates in given conditions are also determined.
\end{abstract}

\section{Introduction}

The potential risk of oil spills is constantly increasing due to the high demand for oil consumption, exploration and climate changes. Since 1999, more than 700 oil spills has been occurred in the world. Every year, a significant amount of oil is spilled into the environment. In Vietnam, according to the report of the Vietnam Administration of Seas and Island from 1987 to 2016, approximately 90 oil spill incidents happened [1].

The cause of the oil spills are often the accidents involving oil exploitation activities of tankers, pipelines, refineries, drilling rigs, and storage facilities (Fig. 1). After a spill, oil components may persist in the environment for long time, even many years that result in serious, immediate and long-term damages to the environment, ecosystems, human activities and socioeconomic.

For example, in August 2006, a 31000 DWT vessel, carrying 23000 tons of oil crashed at the Saigon Port and spilled more than 1500 tons of oil into the environment. After 9 hours, the oil spread over a wide region of 40-50 km downstream of the Saigon River. After 15 days, the affected areas were increased to about 60000 ha, which caused serious damage to the ecosystem and socio-economic activities near the Saigon River because of the ineffective and slow response [1].

In practice, as long as the oil is released into the water, spilled oil should be removed as quickly as possible because it will be weathered after a relatively short period. The oil spreads and floats on the surface with a numerous of physical and chemical changes, hence, in order to facilitate the spill cleanup, special techniques and equipment are required. Practically, the oil cleanup could be realized by using mechanical barriers, skimmers, sorbent materials, dispersing and biological agents, or in-situ burning [2].

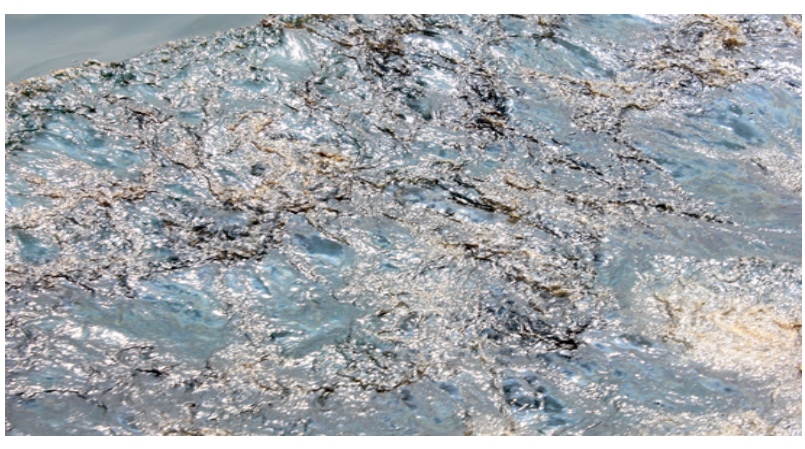

Fig. 1. Oil spill in Quang Ngai, Vietnam (2013).

Among above-mentioned methods, the most common and popular equipment used for oil spill cleanup is using mechanical barriers or oil booms to collect the spilled oil and prevent the slick spreading. The collected, concentrated oil can be recovered using skimming devices.

Although in recent years many different types of booms have been developed, it mainly consists of components such as the freeboard to prevent or reduce splash over, the skirt to prevent or reduce escape of oil under the boom, the flotation by air or some buoyant material and ballast to keep the boom upright in the water and to provide strength to withstand the effects of waves and currents. The advantage of booms over other methods is the simple operation and the absence of adverse environmental effects. However, oil booms can be unsuccessfully performed in recuperating spilled oil depends on its parameters and hydrodynamic conditions. The effectiveness of booms utilization varies from $10 \%$ to $95 \%$ [3]. When oil boom fails, the risk is that the polluted material weight increases significantly multiplied, sometimes by a ratio of 10 [2]. Several failure mechanisms can be occurred such as splashover, submergence, planing failure, entrainment failure,

\footnotetext{
* Corresponding author: chittk@utc.edu.vn
} 
drainage failure, critical accumulation and structural failure (Fig. 2) [4, 5].



Fig. 2. Failure mechanisms of oil booms (Fingas, Mervin).

It can be seen clearly that the cleanup could be negatively affected by even small loss rates. The longer total time will be required to complete the work that leads to many consequences. The leakage from the booms is acceptable if the loss rate is small enough so leaked volume can be removed by the skimmers. However, most of existed works focused on determination the initial failure velocity and structural analysis of booms, e.g. effects of currents, draft and skirt parameters on boom behaviors. It can be found in the studies of authors such as Leibovich S. (1976), Goodman R. H. et al. (1996), Zhang Z. et al. (1999), Amini A. (2009), Xing F. et al. (2011), Shi Y. (2017a, b), Feng X. (2019), etc. [6, 7, 8, 9, 10, 11, 12, 13].

The loss rate has been studied by a few numbers of researchers in order to evaluate the performance of the oil boom in given conditions. Lindenmuth et al. (1970) proposed the simplest entrainment loss relation as below [14]:

$$
\dot{q}_{E}=\beta^{\prime} U_{c} h_{f}
$$

where: $\dot{q}_{E}$ is the oil loss rate; $\mathrm{U}_{\mathrm{c}}$ is the current velocity; $h_{f}$ is the headwave thickness; $\beta$ ' is the unknown entrainment parameter, determined empirically from experiments.

In 1974, Hale L.A. et al. suggested an empirical model for predicting entrainment loss rate based on their results from laboratory tests [15]:

$$
\frac{\dot{q}_{E}}{U_{c} t_{a v}}=4 \cdot 10^{-7}(W e)^{1.74}\left(L / t_{a v}\right)^{0.303}\left(\mu_{o} / \mu_{w}\right)^{0.022}
$$

where: $t_{a v}$ is the average slick thickness, $L$ is the slick length; $\mu_{\mathrm{o}}$ and $\mu_{\mathrm{oi}} \mathrm{l}$ are the oil and water viscosity, respectively.

Zalosh and Jenson (1975) used numerical model to simulate stable or unstable oil slick headwave at different water velocities [16]. They concluded that oil loss rates increase sharply with velocity.

Fannelop (1983) has been estimated the capacity of containment barriers [17]. He focused on the loss mechanisms of two-dimensional and three-dimensional barriers and proposed the correlation for entrainment loss estimates as functions of current speed:

$$
\begin{aligned}
\frac{g \Delta \dot{q}_{E}}{U_{c}^{3}} & =\psi\left(\frac{U_{c}}{(\sigma g \Delta / \rho)^{1 / 4}}\right) \\
\frac{g \Delta \dot{q}_{E}}{U_{c}^{3}} & =\psi\left(\frac{U_{c}}{(\sigma g \Delta / \rho)^{1 / 4}}\right)
\end{aligned}
$$

where: $U_{\mathrm{cr}}$ is the critical velocity for droplets to appear, $\Delta=\left(\rho_{w}-\rho_{o}\right) / \rho_{w}, \sigma$ is the interracial tension. The results showed a difference in loss rate for barriers with different drafts. Even if the loss initiates at similar flow velocities, the loss rate is higher and increases more by increasing the flow velocity in case of a barrier with a shorter draft.

In 2008, Azin Amini proposed a formula for predicting the loss rate in the absence of wave using genetic programming (EPR) as below [18]:

$$
q_{E}=122.79 D V^{2 / 3} \exp \left(22.65 I_{U}\right)+\frac{2.43 I_{U}}{D^{3} V^{7 / 3}}-0.74
$$

where $I_{U}$ the increment of the flow velocity compared to the initial failure velocity, $\mathrm{V}$ the initial oil volume, $\mathrm{D}$ the barrier draft.

In the work of Feng Xing et al. (2011), the loss rate of the oil boom with $0,5 \mathrm{~m}$ draft under weak and aggressive wave conditions is investigated [10]. They concluded that the loss rate increases very rapidly after the initiation of failure in the both cases.

In all above-mentioned studies, the viscous effects of oil to the loss rate of oil booms had not been mentioned in details. Hence, the present study focused on proposing a numerical modelling to provide better understanding of the loss rate of oil booms under different oil viscosities.

\section{Methods}

In this study, software Ansys Fluent has been utilized to develop a two-dimensional two-phase model and simulate the oil-water flow around an oil boom. In FLUENT, the Finite Volume Method (FVM) is used to solve the governing equations for a fluid. FVM based on the control volume formulation. The domain is divided into a number of control volumes, where the variable of interest is located at the centroid of the control volume. Then, the differential form of the governing equations over each control volume is integrated in order to describe the variation of the concerned variable between cell centroids. Interpolation profiles are then assumed. As results, the discretization equation is obtained [19].

For modeling the multiphase flows, there are two approaches which are Lagrangian and Eulerian formulations. In the Lagrangian approach, motion of the 
particles is determined by a steady Lagrangian frame. In an Eulerian approach, the granular phase is treated as another continuum. In this study, the Lagrangian formulation is chosen to modeling the oil-water flow. The fluid flow is first solved, then applied forces to the particles can be determined knowing the flow velocity field. The incremental movement of the particle in each time step is calculated by using the equation of motion for the particles.

At each time step, fluid volume fractions are updated by using the VOF method [20]. The continuity equation for the volume fraction of $q^{\text {th }}$ phase has the following form:

$$
\frac{1}{\rho_{q}}\left[\frac{\partial}{\partial t}\left(\alpha_{q} \rho_{q}\right)+\nabla\left(\alpha_{q} \rho_{q} \vec{v}_{q}\right)=S_{\alpha_{q}}+\sum_{p=1}^{n}\left(\dot{m}_{p q}-\dot{m}_{q p}\right)\right]
$$

Where $\dot{m}_{q p}$ is the mass transfer from phase $q$ to phase $p, \dot{m}_{p q}$ is the mass transfer from phase $p$ to phase $q$.

The turbulence is modeled using the $\mathrm{k}-\varepsilon$ model. The k equation is:

$$
\begin{aligned}
& \frac{\partial}{\partial t}(\rho k)+\frac{\partial}{\partial x_{j}}\left(\rho k u_{j}\right)=\frac{\partial}{\partial x_{j}}\left[\left(\mu+\frac{\mu_{t}}{\mu_{k}}\right) \frac{\partial k}{\partial x_{j}}\right]+ \\
& +G_{k}+G_{b}+\rho \varepsilon-Y_{M}+S_{k}
\end{aligned}
$$

The $\varepsilon$ equation is:

$$
\begin{aligned}
& \frac{\partial}{\partial t}(\rho \varepsilon)+\frac{\partial}{\partial x_{j}}\left(\rho \varepsilon u_{j}\right)=\frac{\partial}{\partial x_{j}}\left[\left(\mu+\frac{\mu_{t}}{\sigma_{i}}\right) \frac{\partial \varepsilon}{\partial x_{j}}\right]+ \\
& +\rho C_{1} S \varepsilon-\rho C_{2} \frac{\varepsilon^{2}}{k+\sqrt{v \varepsilon}}+C_{1 \varepsilon} \frac{\varepsilon}{k} C_{3 \varepsilon} G_{b}+S_{\varepsilon}
\end{aligned}
$$

where $G_{k}$ is the generation of turbulence kinetic energy due to the mean velocity gradients, $G_{b}$ is the generation of turbulence kinetic energy due to buoyancy, $Y_{M}$ is the contribution of the fluctuating dilatation in compressible turbulence to the overall dissipation rate, $C_{2}, C_{1 \varepsilon}$ are constants; $\sigma_{\mathrm{k}}, \sigma_{\varepsilon}$ are the turbulent Prandtl numbers for $\mathrm{k}$ and $\varepsilon$, respectively; $S_{k}, S_{\varepsilon}$ are user-defined source terms.

In the present study, the value of turbulent kinetic energy $\mathrm{k}$ and turbulent dissipation rate $\varepsilon$ can be evaluated according to the following equations:

$$
k=1,5(U I)^{2}
$$

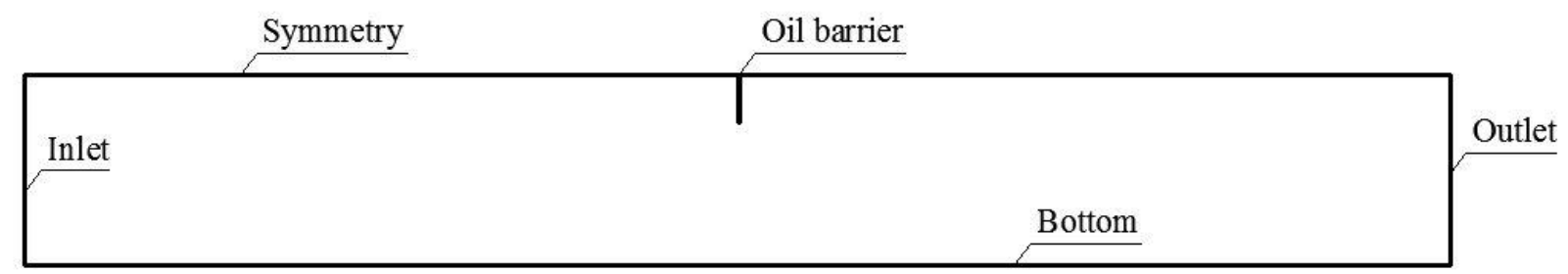

Fig. 3. Boundary conditions.

$$
\varepsilon=C_{\mu}^{3 / 4} \frac{k^{3 / 2}}{l}
$$

where: $I$ is turbulent intensity, $I=0.16(\operatorname{Re})^{1 / /}, R e_{H D}$ is the Reynolds number, $C_{\mu}$ is an empirical constant equal to $0.09, l$ is the turbulence length scale

The geometry and mesh are created using Design Modeler and Meshing tool of Workbench, respectively. Because around the boom, the mean flow can change rapidly and forms shear layers, the fine mesh is applied to capture phase volume fraction fields and high gradient of velocity.

Data from the study carried out by Shi (2018) were adopted as benchmark to clarify the accuracy of the FLUENT numerical model in simulating the flow around the oil boom [12]. The parameters set for the numerical simulations were the same as those used in the experiment. The domain with the length of $9.0 \mathrm{~m}$, the water depth $0.8 \mathrm{~m}$ is established. The boom parameters includes $0.1 \mathrm{~m}$ diameter of floater, $0.1 \mathrm{~m}$ length of skirt. The spilled oil slick is $1 \mathrm{~m}$ long and $0.03 \mathrm{~m}$ thick, placed in front of the boom. The flow velocity is $0.15 \mathrm{~m} / \mathrm{s}$. Oils with different densities and viscosities are modeled to determine the influence of oil viscosity to the capacity of oil boom (Table 1).

Table 1. Oil properties.

\begin{tabular}{|c|c|c|c|}
\hline Oil & $\begin{array}{c}\text { Density } \\
(\mathrm{kg} / \mathrm{m} 3)\end{array}$ & $\begin{array}{c}\text { Viscosity } \\
(\mathrm{kgm} / \mathrm{s})\end{array}$ & $\begin{array}{c}\text { Relative viscosity } \\
\left(\mu_{\mathrm{r}}=\mu_{\mathrm{oil}} / \mu_{\text {water }}\right)\end{array}$ \\
\hline $\begin{array}{c}\text { Industrial gear } \\
\text { lubricant }\end{array}$ & 890 & 1.446 & 1446 \\
\hline Shell Valvata & 915 & 0.915 & 915 \\
\hline Shell gear 85 & 911 & 0.292 & 292 \\
\hline
\end{tabular}

The boundary conditions were set at each boundary of the model, included symmetry at the free-surface, noslip wall at the bottom, velocity inlet at the inlet and pressure outlet at the outlet. The barrier is regarded as non-slip wall.

The residuals of the discretized transport equation were set as value of $1 \times 10^{-6}$ for all variables including pressure, velocity, turbulent kinetic energy and turbulence dissipation rate. For the pressure-velocity, the PISO algorithm (Pressure Implicit with Splitting of Operators) is used. Pressure was discretized using PRESTO Scheme. QUICK Schemes is applied for pressure discretization and for momentum, kinetic energy and dissipation equations. The loss rate is 
calculated as the ratio of oil contained in the upstream of oil boom to the initial oil volume.

\section{Results and Discussion}

To validate the model for describing the oil containment process, analysis of oil slick thickness in front of the oil boom was conducted and the obtained results are compared with the data of Shi et al. (2019). Overall, reasonable agreement could be obtained for Industrial gear lubricant in given conditions (Fig. 3). The oil slicks in these cases have almost the same shape.

Figure 4 shows the shape evolution in numerical models of the oil slick with relative viscosity equal 915 ( $1^{\text {st }}$ case). When the oil approaches the barrier, first, the oil is accumulated near the barrier and then only part of it is escaped under the skirt of the boom. The contained oil is reflected toward the upstream of the barrier. In the zone of the contained oil, three different regions in an oil layer are identified. There is the headwave region at the upstream end of the oil slick with maximum thickness of the oil layer; the middle region contains most of the oil and the near boom region that is affected by a circulation formed behind the oil boom. At the moment when the oil slick is moving to the upstream, a large vortex is formed along the oil layer but then divided into smaller vortexes in the headwave and middle regions.

A zone with high velocity was formed beneath the barrier and near the headwave region. Overall, the velocity beneath the oil layer is higher than in other places (Fig. 5). In this case, $0.17 \mathrm{~m}^{3}$ have leaked to the downstream of the oil boom, hence the loss rate is $57 \%$.

For the $2^{\text {nd }}$ case of oil with the relative viscosity of 292, when accumulated oil reached to the barrier, a local thickening occurred, a crest is forming near the oil boom and then the oil slowly escape just under the boom. The shape evolution of oil slick is shown in Figure 6.

In this case, all of the oil has leaked to barrier downstream. The oil barriers cannot contain the spilled oil and lost its capacity. As we can see from two cases above, as the relative viscosity decreases, the loss rate increases. For the case of oil with relative viscosity of 755 , unfortunately, the oil flow has the same tendency with the $2^{\text {nd }}$ case, all of the oil volume escaped under the barrier to the upstream.

For the case of high relative viscosity $\left(\mu_{\mathrm{r}}=1446\right)$, the shape evolution in numerical models of the oil slick is shown in Figure 7. When the oil approaches the barrier, the slick is shorten and thicken and then



Fig. 4. Numerical results for the oil slick in front of the oil boom.
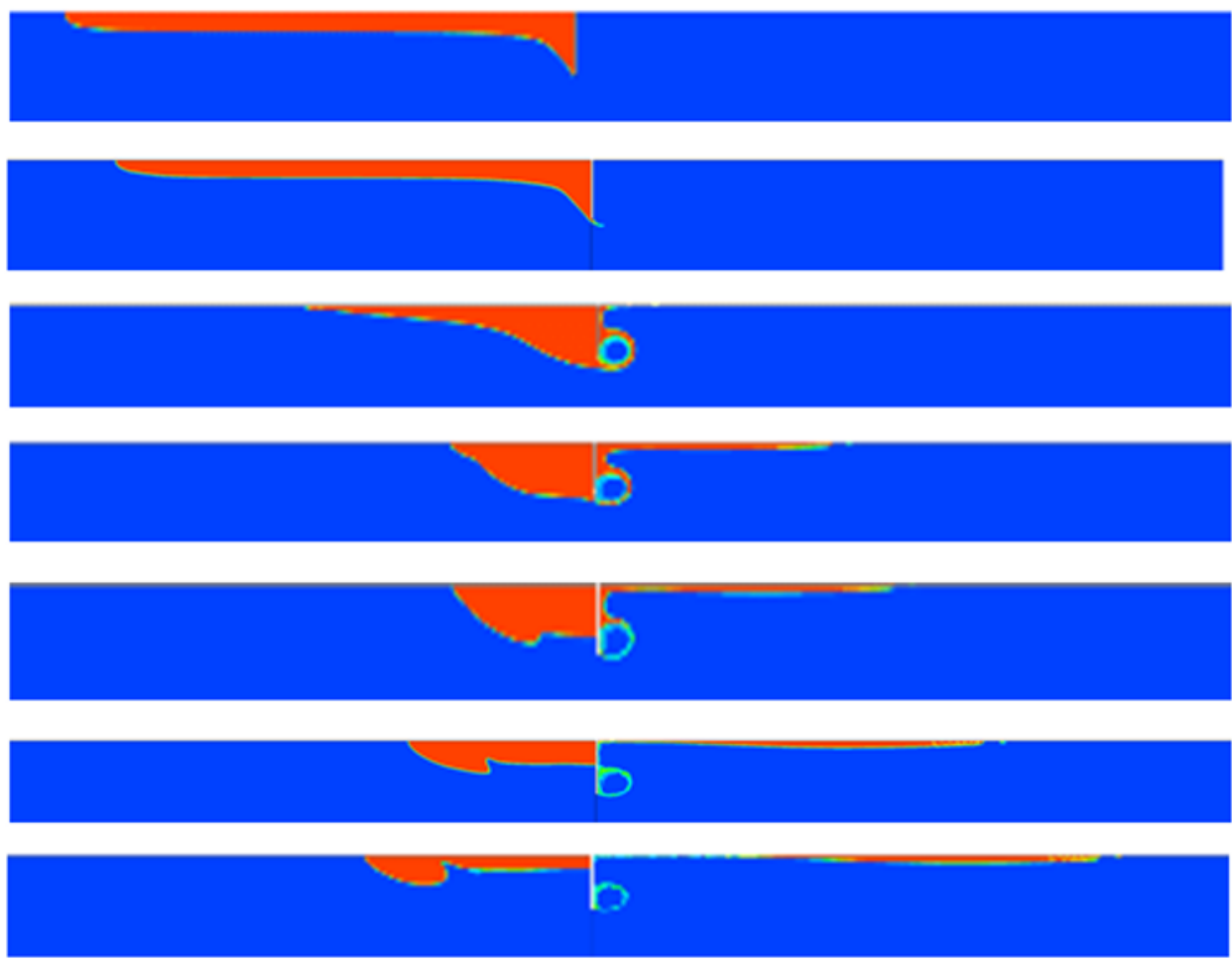

Fig. 5. Shape evolution of oil slick $\left(\mu_{\mathrm{r}}=915\right)$. 
gradually escaped under the skirt of the boom. Unlike the $1^{\text {st }}$ case, the volume of accumulated oil near the boom is not large. Only a very small volume of oil remained at the upper corner of the barrier at the upstream. In the zone of the contained oil, three different regions in an oil layer are identified. There are no headwave regions or vortex that could be observed. So, in this case, although the relative viscosity increases but the loss rate still decreases.

It could be concluded that in given conditions, the viscosity strongly effect the behavior of the oil flow as well as the loss rate of the oil boom. However, more cases should be studied in order to identify the trend of the viscous effects. Based on that, the approach to operate the boom can be adjusted so that the oil concentrated as much as possible in the upstream. In addition, the reasonable range of oil viscosity could be determined that can enhance the effectiveness of the performance of oil.

In this case, all of the oil has leaked to barrier downstream. The oil barriers cannot contain the spilled oil and lost its capacity. As we can see from two cases above, as the relative viscosity decreases, the loss rate increases. For the case of oil with relative viscosity of 755 , unfortunately, the oil flow has the same tendency with the $2^{\text {nd }}$ case, all of the oil volume escaped under the barrier to the upstream.

For the case of high relative viscosity $\left(\mu_{\mathrm{r}}=1446\right)$, the shape evolution in numerical models of the oil slick is shown in Figure 7. When the oil approaches the barrier, the slick is shorten and thicken and then gradually escaped under the skirt of the boom. Unlike the $1^{\text {st }}$ case, the volume of accumulated oil near the boom is not large. Only a very small volume of oil

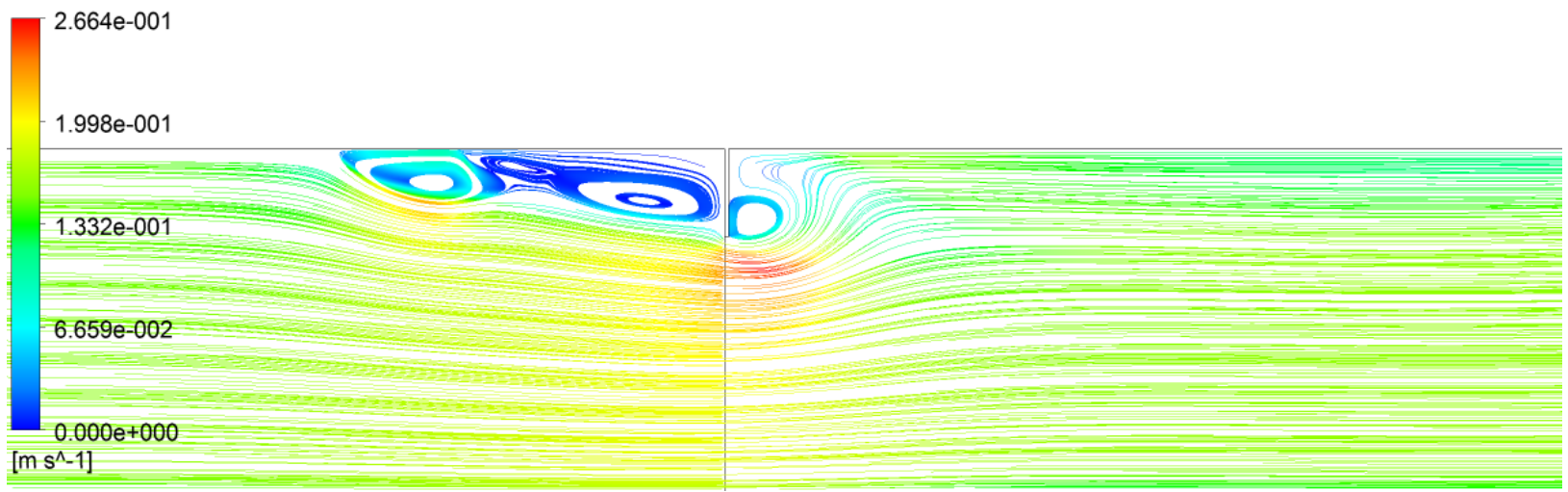

Fig. 6. Velocity streamline around the oil boom $\left(\mu_{\mathrm{r}}=915\right)$.
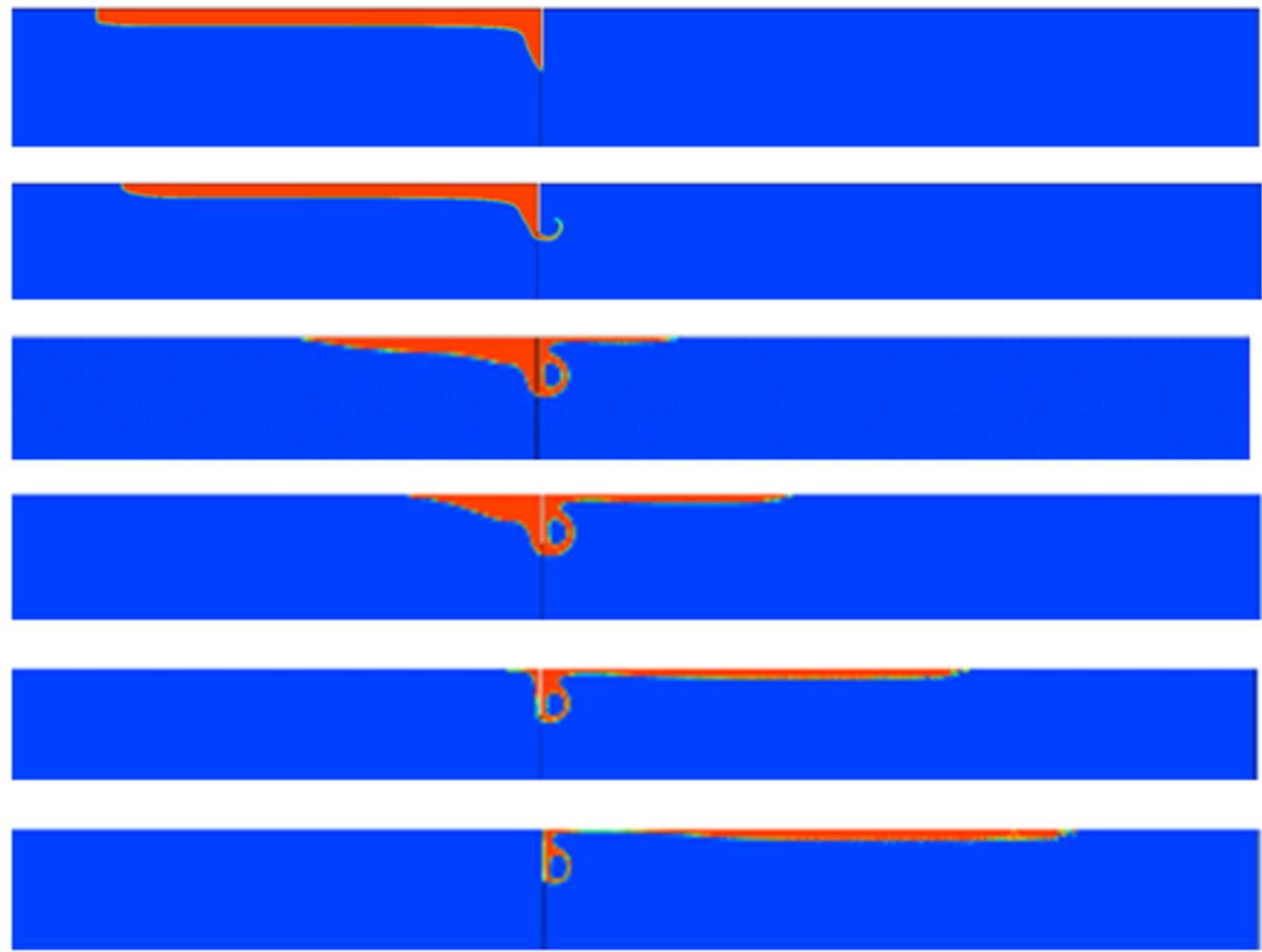

Fig. 7. Shape evolution of oil slick $\left(\mu_{\mathrm{r}}=292\right)$. 

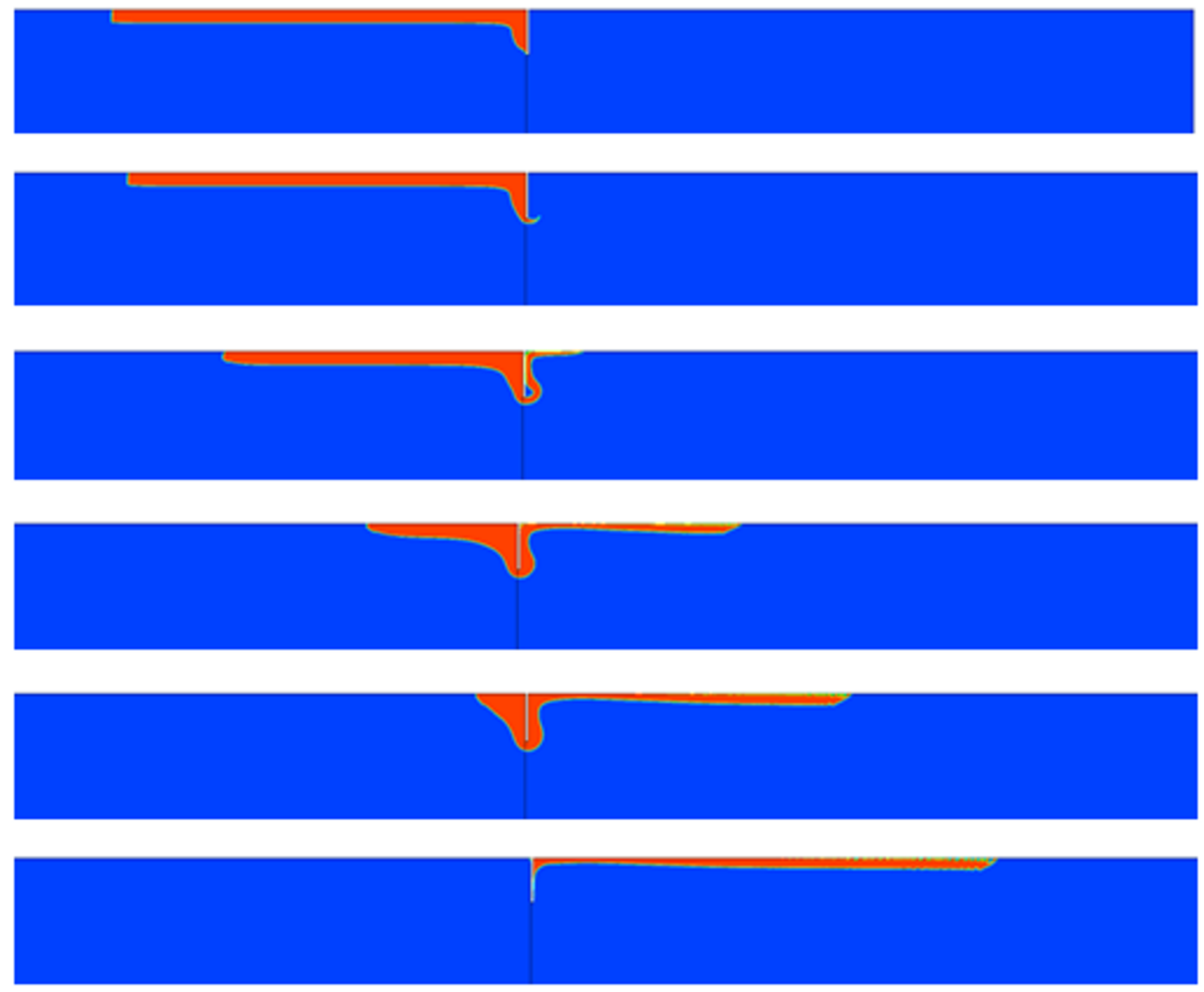

Fig. 8. Shape evolution of oil slick $\left(\mu_{\mathrm{r}}=1446\right)$.

remained at the upper corner of the barrier at the upstream. In the zone of the contained oil, three different regions in an oil layer are identified. There are no headwave regions or vortex that could be observed. So, in this case, although the relative viscosity increases but the loss rate still decreases.

It could be concluded that in given conditions, the viscosity strongly effect the behavior of the oil flow as well as the loss rate of the oil boom. However, more cases should be studied in order to identify the trend of the viscous effects. Based on that, the approach to operate the boom can be adjusted so that the oil concentrated as much as possible in the upstream. In addition, the reasonable range of oil viscosity could be determined that can enhance the effectiveness of the performance of oil.

\section{Conclusions}

Mechanical oil booms are key equipment that used to contain or divert the oil slick. In the present study, a two dimensional numerical model has been applied to investigate the viscous effects of oil to the capacity of oil booms. As results, the viscosity strongly effect the behavior of the oil flow as the shape of the oil slick around the oil boom gradually varies with viscosities even under the same hydrodynamic conditions. The loss rates are different as well. When the relative viscosity decreases, the loss rate might either increase or decrease.

The goal of this study is to obtain a better knowledge of the performance of oil booms. More cases should be studied in future in order to evaluate the influence of oil viscosity to the capacity of oil booms. Based on that, the acceptable parameters of oil booms could be chosen so that can meet the requirements in given conditions for the given viscosity of oil in order to enhance the effectiveness of the performance of oil booms.

\section{References}

P. Nguyen, Eur. J. of Eng. Res. and Sci., 3, 7 (Jul. 2018), pp. 1-4 (2018)

M. Fingas, Oil Spill Science and Technology. Amsterdam: Elsevier (2017)

K. Wong, E. Barin, Spill Sci. and Tech. Bul., 8 (5-6), pp. 509-520, (2003)

D. Violeau, C. Buvat, K. Abed-Meraim, E. de Nanteuil, Coas. Eng., 54 (12), pp 895-913 (2007). 
M. Fingas, Oil Spill Sci. Technol, Prevention, Response and Cleanup, Burlington, MA: Gulf Professional Publishing, pp. 435-535, (2011)

S. Leibovich, J. of Flu. Eng., 98, pp. 93-108 (1976)

R.H. Goodman, H.M. Brown, C.F. An, R. D.Rowe, Spill Sci. and Tech. Bull., vol 3(4), pp. 213-216 (1996).

Z. Zhang, S. Cheng, Lihan, C.F. An, Act. Sci. Cir., 6, pp. 604-609 (1999)

A. Amini, A. J. Schleiss, Eng. Appl. of Comp. Flu. Mech., 3, pp 207-219 (2009).

F. Xing, W. Wanqing, W. Wenfeng, Proc. Env. Sci., vol. 8, No. 0, pp 40-47 (2011)

Y. Shi, S. Li, H. Zhang, S. Peng, H.Chen, Appl. Ocean. Res., 69: pp. 38-52 (2017)

Y. Shi, S. Li, H. Zhang, S. Peng, H. Chen, R. Zhou, T. Mao, J. Ocean Univ. China, 16 (4), pp. 602-608 (2017)

X. Feng, Y. Shi. J. of Eng. Mech., 145 (2019).

W.T. Lindenmuth, E.R. Miller, C.C.Hsu, US Coast Guard Report, (714102/A/008) (1970)

R.K. Agrawal, L.A. Hale, Offshore Technology Conference, Texas, USA, pp. 461-466 (1974)

R.G. Zalosh , D.S. Jenson, Symposium on Fluid mechanics in the Petroleum Industry, ASME, pp. 17-27 (1975)

T.K. Fannelop, Appl. Oc. Res., 5(2): pp.80-92 (1983)

A. Amini, E. Bollaert, J.L. Boillat, A.J. Schleiss, Oc. Eng., 35(14-15): pp.1479-1491 (2008)

ANSYS Fluent Theory Guide. Release 15.0. ANSYS, Inc. November. 814 p. (2013)

Hirt C.W., Nichols B.D, J. Comp. Phys., 39(1), pp. 201225 (1981). 